\title{
ON BOUNDARY VALUE PROBLEMS WITH PRESCRIBED NUMBER OF ZEROES OF SOLUTIONS
}

\author{
A. RONTÓ, M. RONTÓ, AND N. SHCHOBAK
}

Received 17 May, 2017

\begin{abstract}
We show how appropriate parametrisation technique and successive approximations can help to investigate solutions of Emden-Fowler type equations with a given number of zeroes. The technique can be efficiently applied for more general equations with non-linearities involving absolute value signs and various types of boundary conditions.
\end{abstract}

2010 Mathematics Subject Classification: 34B15

Keywords: Emden-Fowler equation, model-type boundary conditions, successive approximations, prescribed number of zeroes

\section{INTRODUCTION}

The need to construct solutions with a prescribed number of zeroes arises quite often in the theory of ordinary differential equations (see, e. g., [2, 3, 9, 11, 12, 14] and references therein). Due to the overall complexity of the problem, it is natural to observe that the proof of the existence of such solutions is usually of qualitative character and is seldom constructive. One can cite considerably less works where the solution in question is found in a straightforward way.

The aim of this note is to demonstrate that the numerical-analytic techniques with interval divisions [17-19] can serve this purpose. Being far from claiming that, in this way, a complete description is possible (of course, every approach has its pros and contras), we should like to show that this idea provides one a rather general "machinery" for practical finding solutions with a given number of sign changes.

We demonstrate the approach on a particular case of the Emden-Fowler type equation

$$
u^{\prime \prime}(t)=-q(t)|u(t)|^{3} u(t)+r(t), \quad t \in[a, b],
$$

where $q(t)>0$ for all $t \in[a, b]$, for which the question mentioned has long been of interest. Note that, despite their seemingly simple form, these equations are known to exibit very complicated behaviour of solutions [7]. For this class of equations, the existence of solutions with a given number of zeroes is studied, in particular, in $[1,5,13]$. 
The Nehari theory for Emden-Fowler type equations (1.1) provides a way to detect the existence of solutions with prescribed number of zeros [4-6,10,14]. For instance, in the case of the Dirichlet boundary value problem

$$
\begin{gathered}
u^{\prime \prime}(t)=-q(t)(u(t))^{3}, \quad t \in[-1,1], \\
u(-1)=0, \quad u(1)=0,
\end{gathered}
$$

where $q$ is the step function

$$
q(t)= \begin{cases}2 & \text { if } t \in[-1,-1+\alpha] \\ 0 & \text { if } t \in(-1+\alpha, 1-\alpha) \\ 2 & \text { if } t \in[1-\alpha, 1]\end{cases}
$$

and $\alpha$ is chosen in a specific way, the Nehari theory gives three positive solutions of (1.2). In [4], the authors construct three positive solutions of problem (1.2) for $q(t)=2 /(1+h \xi(t))^{6}$ with an $h>0$ and the " $\wedge$-shaped" piecewise linear $\xi$,

$$
\xi(t)= \begin{cases}1+t & \text { for }-1 \leq t<0, \\ 1-t & \text { for } 0 \leq t \leq 1\end{cases}
$$

The methods used to construct the solution usually strongly depend on the specific form of the equation. In particular, the approach of [4] is based on the explicit use of the lemniscate functions sl $t$ and $\mathrm{cl} t$, i. e., the respective solutions of the initial value problems $u(0)=0, u^{\prime}(0)=1$ and $u(0)=1, u^{\prime}(0)=0$ for the equation $u^{\prime \prime}=-2 u^{3}$.

Motivated, in particular, by the work [4] on the above-mentioned problem of form (1.2), we show here how parametrisation techniques can be used to construct solutions of this kind of equations. We describe the approach for the Dirichlet problem

$$
\begin{aligned}
u^{\prime \prime}(t)= & g\left(t, u(t), u^{\prime}(t)\right), \quad t \in[a, b], \\
& u(a)=0, \quad u(b)=0,
\end{aligned}
$$

focusing on several concrete Emden-Fowler type equations of form (1.1), which quite well illustrates the idea (the techniques are, however, not limited to this case). Depending on the choice of $q$ and $r$ in (1.1), we study several cases where sign-constant and sign-changing solutions are detected.

We use here the numerical-analytic approach from [19], which allows one to constuct approximate the solutions of problem (4.4), (4.5) and, moreover, rigorously prove the existence of an exact solutions by using the results of computation. More details on the techniquees discussed and used here can be found, e. g., in [15-18]. We describe how these techniques can be efficiently used to find either sign-constant or sign-changing solutions. 


\section{THE METHOD}

Consider the two-point problem

$$
\begin{aligned}
& u_{1}^{\prime}(t)=f_{1}\left(t, u_{1}(t), u_{2}(t)\right), \\
& u_{2}^{\prime}(t)=f_{2}\left(t, u_{1}(t), u_{2}(t)\right), \quad t \in[a, b], \\
& u_{1}(a)=0, \quad u_{1}(b)=0 .
\end{aligned}
$$

The Dirichlet problem (1.3), (1.4) obviously corresponds to the case where system (2.1), (2.2) has the form

$$
\begin{aligned}
& u_{1}^{\prime}(t)=u_{2}(t), \\
& u_{2}^{\prime}(t)=g\left(t, u_{1}(t), u_{2}(t)\right), \quad t \in[a, b] .
\end{aligned}
$$

System (2.1), (2.2), (1.4) will be treated using parametrisation technique by "freezing" the values of the solution at suitable time instants.

\subsection{Auxiliary problems}

Let $t_{1}$ be a point from $(a, b)$. Consider the two-point problems

$$
\begin{aligned}
& x_{1}^{\prime}(t)=f_{1}\left(t, x_{1}(t), x_{2}(t)\right) \\
& x_{2}^{\prime}(t)=f_{2}\left(t, x_{1}(t), x_{2}(t)\right), \quad t \in\left[a, t_{1}\right], \\
& x_{1}(a)=0, \quad x_{2}(a)=z_{2}, \\
& x_{1}\left(t_{1}\right)=\eta_{1}, \quad x_{2}\left(t_{1}\right)=\eta_{2},
\end{aligned}
$$

and

$$
\begin{aligned}
& y_{1}^{\prime}(t)=f_{1}\left(t, y_{1}(t), y_{2}(t)\right), \\
& y_{2}^{\prime}(t)=f_{2}\left(t, y_{1}(t), y_{2}(t)\right), \quad t \in\left[t_{1}, b\right], \\
& y_{1}\left(t_{1}\right)=\eta_{1}, \quad y_{2}\left(t_{1}\right)=\eta_{2}, \\
& y_{1}(b)=0, \quad y_{2}(b)=\lambda_{2},
\end{aligned}
$$

where $z_{2}, \eta_{1}, \eta_{2}$ and $\lambda_{2}$ are some real parameters. The form of problems (2.6), (2.7) and (2.8), (2.9) suggests that the functions

$$
u_{i}(t):= \begin{cases}x_{i}(t) & \text { for } t \in\left[a, t_{1}\right] \\ y_{i}(t) & \text { for } t \in\left[t_{1}, b\right]\end{cases}
$$

where $i=1,2$, can be used to construct a solution of the original problem (1.3). Obviously, $\left(u_{1}, u_{2}\right)$ defined by (2.10) is a $C^{2}$ solution of (1.3) if and only if

$$
x_{1}\left(t_{1}\right)=y_{1}\left(t_{1}\right), \quad x_{2}\left(t_{1}\right)=y_{2}\left(t_{1}\right) .
$$

It is thus natural to study continuously differentiable solutions of the boundary value problems (2.6), (2.7) and (2.8), (2.9) for which the continuous "gluing" property (2.11) holds. 


\subsection{Notation and symbols, some subsidary statements}

In the sequel, for any vector $x=\operatorname{col}\left(x_{1}, x_{2}\right) \in \mathbb{R}^{2}$, the obvious notation $|x|=$ $\operatorname{col}\left(\left|x_{1}\right|,\left|x_{2}\right|\right)$ is used and the inequalities between vectors are understood componentwise. Maxima and minima of vector-valued functions are likewise understood componentwise.

The symbol $\mathbb{1}_{2}$ stands for the unit matrix of dimension $2, r(K)$ denotes the maximal, in modulus, eigenvalue of a square matrix $K$.

For any non-negative vector $\rho \in \mathbb{R}^{2}$, by a componentwise $\rho$-neighbourhood of a point $z \in \mathbb{R}^{2}$, we understand the set

$$
O_{\rho}(z):=\left\{\xi \in \mathbb{R}^{2}:|\xi-z| \leq \rho\right\} .
$$

Similarly, the componentwise $\rho$-neighbourhood of a set $\Omega \subset \mathbb{R}^{2}$ is defined as

$$
O_{\rho}(\Omega):=\bigcup_{\xi \in \Omega} O_{\rho}(\xi)
$$

For given two bounded sets $D_{0} \subset \mathbb{R}^{2}$ and $D_{1} \subset \mathbb{R}^{2}$, introduce the set

$$
\mathscr{B}\left(D_{0}, D_{1}\right):=\left\{(1-\theta) z+\theta \eta: z \in D_{0}, \eta \in D_{1}, \theta \in[0,1]\right\} .
$$

For any $a_{0}, b_{0}, a \leq a_{0}<b_{0} \leq b$, and a continuous function $f:[a, b] \times D \rightarrow \mathbb{R}^{k}$, $k \in\{1,2\}, D \subset \mathbb{R}^{2}$, we put

$$
\delta_{\left[a_{0}, b_{0}\right], D}(f):=\max _{(t, x) \in\left[a_{0}, b_{0}\right] \times D} f(t, x)-\min _{(t, x) \in\left[a_{0}, b_{0}\right] \times D} f(t, x)
$$

and $\delta_{\left[a_{0}, b_{0}\right], \mathbb{R}^{n}}(f):=\delta_{\left[a_{0}, b_{0}\right]}(f)$.

For a non-negative $2 \times 2$ square matrix $K$, the notation $f \in \operatorname{Lip}_{K}\left(\Omega,\left[a_{0}, b_{0}\right]\right)$ means that the componentwise inequality

$$
\left|f\left(t, u_{1}\right)-f\left(t, u_{2}\right)\right| \leq K\left|u_{1}-u_{2}\right|
$$

holds for all $t \in\left[a_{0}, b_{0}\right]$ and $u_{1}, u_{2}$ from $\Omega$.

Let us put

$$
\alpha_{1}\left(t, a_{0}, b_{0}\right):=2\left(t-a_{0}\right)\left(1-\frac{t-a_{0}}{b_{0}-a_{0}}\right), \quad t \in\left[a_{0}, b_{0}\right],
$$

for any $a_{0}, b_{0}, a \leq a_{0}<b_{0} \leq b$.

\subsection{Iterations for auxiliary problems}

Let $D_{a}, D_{b}$, and $D_{t_{1}}$ be certain fixed closed bounded sets in $\mathbb{R}^{2}$. The notation reflects the fact that we shall look for solutions $u$ with $u(s) \in D_{s}, s \in\left\{a, t_{1}, b\right\}$. In view of the form of the auxiliary boundary conditions (2.7), (2.9), it is natural to suppose that

$$
D_{a} \cup D_{b} \subset\{0\} \times \mathbb{R} .
$$


For any non-negative vectors $\rho_{0}$ and $\rho_{1}$, put

$$
\Omega_{0}\left(\rho_{0}\right):=O_{\rho_{0}}\left(\mathscr{B}\left(D_{a}, D_{t_{1}}\right)\right), \quad \Omega_{1}\left(\rho_{1}\right):=O_{\rho_{1}}\left(\mathscr{B}\left(D_{t_{1}}, D_{b}\right)\right) .
$$

According to (2.13) and (2.14), $\Omega_{0}\left(\rho_{0}\right)$ and $\Omega_{1}\left(\rho_{1}\right)$ are, in fact, neighbourhoods of the sets of straight line segments joining, respectively, $D_{a}$ with $D_{t_{1}}$ and $D_{t_{1}}$ with $D_{b}$. Conditions on the equation will be imposed in the sequel on these wider sets with suitable $\rho_{0}$ and $\rho_{1}$ (see Assumptions $1-3$ below).

The auxiliary two-point boundary value problems (2.6), (2.7) and (2.8), (2.9) will be treated by using iteration schemes suitable for the case of separated two-point conditions. More precisely, for any $m=0,1, \ldots$, put

$$
\begin{aligned}
x_{0}(t, z, \eta)= & \left(1-\frac{t-a}{t_{1}-a}\right) z+\frac{t-a}{t_{1}-a} \eta, \\
x_{m+1}(t, z, \eta)= & x_{0}(t, z, \eta) \\
& +\int_{a}^{t} f\left(s, x_{m}(s, z, \eta)\right) d s-\frac{t-a}{t_{1}-a} \int_{a}^{t_{1}} f\left(\tau, x_{m}(s, z, \eta)\right) d s
\end{aligned}
$$

for $t \in\left[a, t_{1}\right]$ and

$$
\begin{aligned}
y_{0}(t, \eta, \lambda)= & \left(1-\frac{t-t_{1}}{b-t_{1}}\right) \eta+\frac{t-t_{1}}{b-t_{1}} \lambda, \\
y_{m+1}(t, \eta, \lambda)= & y_{0}(t, \eta, \lambda) \\
& +\int_{t_{1}}^{t} f\left(s, y_{m}(s, \eta, \lambda)\right) d s-\frac{t-t_{1}}{b-t_{1}} \int_{t_{1}}^{b} f\left(s, y_{m}(s, \eta, \lambda)\right) d s
\end{aligned}
$$

for $t \in\left[t_{1}, b\right]$. Here, $f=\operatorname{col}\left(f_{1}, f_{2}\right)$. The values $z \in D_{a}, \eta \in D_{t_{1}}, \lambda \in D_{b}$ in (2.19) (2.22) are considered as parameters.

It is clear that, for any fixed $t_{1}$, the graphs of $x_{0}(\cdot, z, \eta)$ and $y_{0}(\cdot, \eta, \lambda)$ given by (2.19), (2.21) are the straight line segments joining the points $(a, z),\left(t_{1}, \eta\right)$ and $(b, \lambda)$. Moreover, the following proposition is obvious from (2.19)-(2.22).

Proposition 1. All the functions $\left\{x_{m}(\cdot, z, \eta): m \geq 0\right\}$ (resp., $\left\{y_{m}(\cdot, z, \eta): m \geq 0\right\}$ ) are continuously differentiable on $\left[a, t_{1}\right]$ (resp., $\left.\left[t_{1}, b\right]\right)$ and satisfy conditions (2.7) (resp., (2.9)).

Let us assume the following conditions.

Assumption 1. There exist non-negative vectors $\rho_{0}$ and $\rho_{1}$ such that

$$
\rho_{0} \geq \frac{t_{1}-a}{4} \delta_{\left[a, t_{1}\right], \Omega_{0}\left(\rho_{0}\right)}(f), \quad \rho_{1} \geq \frac{b-t_{1}}{4} \delta_{\left[t_{1}, b\right], \Omega_{1}\left(\rho_{1}\right)}(f) .
$$

Recall that $\delta_{\left[a, t_{1}\right], \Omega_{0}\left(\rho_{0}\right)}(f)$ and $\delta_{\left[t_{1}, b\right], \Omega_{1}\left(\rho_{1}\right)}(f)$ are given by (2.15), whereas $\Omega_{0}\left(\rho_{0}\right)$ and $\Omega_{1}\left(\rho_{1}\right)$ are sets (2.18).

Assumption 2. There exist non-negative $2 \times 2$ matrices $K_{0}$ and $K_{1}$ such that $f \in$ $\operatorname{Lip}_{K_{0}}\left(\Omega_{0}\left(\rho_{0}\right),\left[a, t_{1}\right]\right)$ and $f \in \operatorname{Lip}_{K_{1}}\left(\Omega_{1}\left(\rho_{1}\right),\left[t_{1}, b\right]\right)$. 
The meaning of these two assumptions is that $f$ is Lipschitzian on a sufficiently wide neighbourhood of the set which is expected to contain the values of potential solutions.

Assumption 3. The maximal in modulus eigenvalues of the matrices $K_{0}, K_{1}$ satisfy the inequalities

$$
r\left(K_{0}\right)<\frac{10}{3\left(t_{1}-a\right)}, \quad \quad r\left(K_{0}\right)<\frac{10}{3\left(b-t_{1}\right)} .
$$

Under these assumptions, sequences (2.20) and (2.22) are uniformly convergent.

Theorem 1. Let Assumptions $1-3$ hold. Then, for all fixed $z \in D_{a}, \eta \in D_{t_{1}}$ and $\lambda \in D_{b}$ :

1. For any $m=0,1, \ldots$,

$\left\{x_{m}(t, z, \eta): t \in\left[a, t_{1}\right]\right\} \subset \Omega_{0}\left(\rho_{0}\right), \quad\left\{y_{m}(t, z, \eta): t \in\left[t_{1}, b\right]\right\} \subset \Omega_{1}\left(\rho_{1}\right)$.

2. The limit functions $x_{\infty}(t, z, \eta):=\lim _{m \rightarrow \infty} x_{m}(t, z, \eta)$ and $y_{\infty}(t, \eta, \lambda):=$ $\lim _{m \rightarrow \infty} y_{m}(t, \eta, \lambda)$ exist uniformly on $\left[a, t_{1}\right]$ and $\left[t_{1}, b\right]$ respectively.

3. $x_{\infty}(\cdot, z, \eta)$ and $y_{\infty}(\cdot, z, \eta)$ satisfy the boundary conditions (2.7), (2.9) and the initial conditions

$$
x_{\infty}(a, z, \eta)=z, \quad y_{\infty}\left(t_{1}, \eta, \lambda\right)=\eta .
$$

4. The functions $x_{\infty}(\cdot, z, \eta)$ and $y_{\infty}(\cdot, \eta, \lambda)$ are unique continuously differentiable solutions of the problems

$$
\begin{aligned}
& x^{\prime}(t)=f(t, x(t))+\frac{1}{t_{1}-a} \Delta^{0}(z, \eta), \quad t \in\left[a, t_{1}\right], \\
& x(a)=z, \quad x\left(t_{1}\right)=\eta,
\end{aligned}
$$

and

$$
\begin{aligned}
& y^{\prime}(t)=f(t, y(t))+\frac{1}{b-t_{1}} \Delta^{1}(z, \eta), \quad t \in\left[t_{1}, b\right], \\
& y\left(t_{1}\right)=\eta, \quad y(b)=\lambda,
\end{aligned}
$$

respectively, where

$$
\begin{aligned}
& \Delta^{0}(z, \eta):=\eta-z-\int_{a}^{t_{1}} f\left(s, x_{\infty}(s, z, \eta)\right) d s, \\
& \Delta^{1}(\eta, \lambda):=\lambda-\eta-\int_{t_{1}}^{b} f\left(s, y_{\infty}(s, \eta, \lambda)\right) d s
\end{aligned}
$$

for all $z \in D_{a}, \eta \in D_{t_{1}}, \lambda \in D_{b}$.

5. The estimates

$$
\left|x_{\infty}(t, z, \eta)-x_{m}(t, z, \eta)\right| \leq \frac{5}{9} \alpha_{1}\left(t, a, t_{1}\right) Q_{0}^{m}\left(\mathbb{1}_{n}-Q_{0}\right)^{-1} \delta_{\left[a, t_{1}\right], \Omega_{0}\left(\rho_{0}\right)}(f)
$$




$$
\begin{aligned}
& \text { for } t \in\left[a, t_{1}\right], m \geq 0 \text { and } \\
& \left|y_{\infty}(t, \eta, \lambda)-y_{m}(t, \eta, \lambda)\right| \leq \frac{5}{9} \alpha_{1}\left(t, t_{1}, b\right) Q_{1}^{m}\left(\mathbb{1}_{n}-Q_{1}\right)^{-1} \delta_{\left[t_{1}, b\right], \Omega_{1}\left(\rho_{1}\right)}(f) \\
& \text { for } t \in\left[t_{1}, b\right], m \geq 0, \text { hold, where } Q_{0}:=\frac{3}{10}\left(t_{1}-a\right) K_{0}, Q_{1}:=\frac{3}{10}\left(b-t_{1}\right) K_{1}, \\
& \text { and the functions } \alpha_{1}\left(\cdot, a, t_{1}\right), \alpha_{1}\left(\cdot, t_{1}, b\right) \text { are defined according to }(2.16) .
\end{aligned}
$$

According to this theorem, the limits of sequences (2.19), (2.20) and (2.21), (2.22) can be interpreted as solutions of the Cauchy problems (2.26), (2.27) and (2.28), (2.29) involving the free parameters $z, \eta$, and $\lambda$. The proof is carried out by analogy to Theorems 1 and 2 from [21].

\subsection{Relation of $x_{\infty}(\cdot, z, \eta)$ and $y_{\infty}(\cdot, \eta, \lambda)$ to the original problem}

It follows from Theorem 1 that the functions $D_{a} \times D_{t_{1}} \ni(z, \eta) \mapsto \Delta^{0}(z, \eta)$ and $D_{t_{1}} \times D_{b} \ni(\eta, \lambda) \mapsto \Delta^{1}(\eta, \lambda)$ are well defined.

Theorem 2. Under the assumptions of Theorem 1, the function

$$
u(t):= \begin{cases}x_{\infty}(t, z, \eta) & \text { for } t \in\left[a, t_{1}\right] \\ y_{\infty}(t, \eta, \lambda) & \text { for } t \in\left[t_{1}, b\right]\end{cases}
$$

is a $C^{2}$ solution of the original boundary value problem (2.1), (2.2), (2.3) if and only if the parameters $(z, \eta, \lambda)$ satisfy the determining equations

$$
\Delta^{0}(z, \eta)=0, \quad \Delta^{1}(\eta, \lambda)=0 .
$$

This statement is proved by analogy to [22, Theorem 3]. Note that, by virtue of the given Dirichlet boundary conditions, the first component of $z$ and $\lambda$ involved in (2.33) is equal to zero. Therefore, (2.33) is a system of four equations

$$
\Delta^{0}\left(\left(\begin{array}{c}
0 \\
z_{2}
\end{array}\right),\left(\begin{array}{c}
\eta_{1} \\
\eta_{2}
\end{array}\right)\right)=0, \quad \Delta^{1}\left(\left(\begin{array}{c}
\eta_{1} \\
\eta_{2}
\end{array}\right),\left(\begin{array}{c}
0 \\
\lambda_{2}
\end{array}\right)\right)=0
$$

with respect to four unknown scalar variables $z_{2}, \eta_{1}, \eta_{2}$ and $\lambda_{2}$.

The next statement establishes that the system of determining equations (2.33) detects all solutions of the boundary value problem (4.4), (4.5) with graphs contained in the given domain.

Theorem 3. Assume that conditions of Theorem 1 are satisfied. If equations (2.33) hold for some $z=\operatorname{col}\left(0, z_{2}^{*}\right) \in D_{a}, \eta=\operatorname{col}\left(\eta_{1}^{*}, \eta_{2}^{*}\right) \in D_{t_{1}}, \lambda=\operatorname{col}\left(0, \lambda_{2}^{*}\right) \in D_{b}$, then function (2.32) is a solution of the boundary value problem (2.1)-(2.3) satisfying the conditions

$$
u(a)=\left(\begin{array}{c}
0 \\
z_{2}^{*}
\end{array}\right), \quad u\left(t_{1}\right)=\left(\begin{array}{c}
\eta_{1}^{*} \\
\eta_{2}^{*}
\end{array}\right), \quad u(b)=\left(\begin{array}{c}
0 \\
\lambda_{2}^{*}
\end{array}\right)
$$


Conversely, if problem (2.1)-(2.3) has a solution $u(\cdot)$ such that $u(a) \in D_{a}, u\left(t_{1}\right) \in$ $D_{t_{1}}, u(b) \in D_{b}$, and ${ }^{1}$

$$
\left\{u(t): t \in\left[a, t_{1}\right]\right\} \subset \Omega_{0}\left(\rho_{0}\right), \quad\left\{u(t): t \in\left[t_{1}, b\right]\right\} \subset \Omega_{1}\left(\rho_{1}\right),
$$

then it necessarily has form (2.32) with

$$
z=\left(\begin{array}{c}
0 \\
u_{2}(a)
\end{array}\right), \quad \eta=u\left(t_{1}\right), \quad \lambda=\left(\begin{array}{c}
0 \\
u_{2}(b)
\end{array}\right)
$$

and values (2.36) satisfy the system of determining equations (2.33).

Proof. The proof is similar to that of [22, Theorem 4].

The solvability of the determining system (2.33) can be studied by analogy to [20] using topological degree methods [8] based on properties of approximate determining functions

$$
\begin{aligned}
& \Delta_{m}^{0}(z, \eta):=\eta-z-\int_{a}^{t_{1}} f\left(s, x_{m}(s, z, \eta)\right) d s, \\
& \Delta_{m}^{1}(\eta, \lambda):=\lambda-\eta-\int_{t_{1}}^{b} f\left(s, y_{m}(s, \eta, \lambda)\right) d s
\end{aligned}
$$

for fixed $m$. Formulae (2.37), unlike (2.30), (2.31), involve only known functions and the equations

$$
\Delta_{m}^{0}(z, \eta)=0, \quad \Delta_{m}^{1}(\eta, \lambda)=0
$$

form an approximation to the determining system (2.33). For the Dirichlet problem, similarly to (2.34), system (2.38) is rewritten as

$$
\Delta_{m}^{0}\left(\left(\begin{array}{c}
0 \\
z_{2}
\end{array}\right),\left(\begin{array}{c}
\eta_{1} \\
\eta_{2}
\end{array}\right)\right)=0, \quad \Delta_{m}^{1}\left(\left(\begin{array}{c}
\eta_{1} \\
\eta_{2}
\end{array}\right),\left(\begin{array}{c}
0 \\
\lambda_{2}
\end{array}\right)\right)=0 .
$$

\subsection{Approximation of a solution}

If $(\tilde{z}, \tilde{\eta}, \tilde{\lambda}) \in D_{a} \times D_{t_{1}} \times D_{b}$ is a root of the approximate determining system (2.38) for a certain fixed $m$, then the function

$$
U_{m}(t)= \begin{cases}x_{m}(t, \tilde{z}, \tilde{\eta}) & \text { for } t \in\left[a, t_{1}\right], \\ y_{m}(t, \tilde{\eta}, \tilde{\lambda}) & \text { for } t \in\left[t_{1}, b\right],\end{cases}
$$

defined according to (2.20), (2.22) can be regarded as the $m$ th approximation to a solution of problem (2.4), (2.5), (2.3). This is justified by the estimates

$$
\left|x_{\infty}(t, \tilde{z}, \tilde{\eta})-x_{m}(t, \tilde{z}, \tilde{\eta})\right| \leq \frac{5}{9} \alpha_{1}\left(t, a, t_{1}\right) Q_{0}^{m}\left(\mathbb{1}_{n}-Q_{0}\right)^{-1} \delta_{\left[a, t_{1}\right], \Omega_{0}\left(\rho_{0}\right)}(f)
$$

\footnotetext{
${ }^{1}$ Recall that $\rho_{0}, \rho_{1}$ are the values involved in Assumption 1 of Theorem 1 and $\Omega_{0}\left(\rho_{0}\right), \Omega_{1}\left(\rho_{1}\right)$ are the corresponding sets (2.18).
} 
for $t \in\left[a, t_{1}\right], m \geq 0$, and

$$
\left|y_{\infty}(t, \tilde{\eta}, \tilde{\lambda})-y_{m}(t, \tilde{\eta}, \tilde{\lambda})\right| \leq \frac{5}{9} \alpha_{1}\left(t, t_{1}, b\right) Q_{1}^{m}\left(\mathbb{1}_{n}-Q_{1}\right)^{-1} \delta_{\left[t_{1}, b\right], \Omega_{1}\left(\rho_{1}\right)}(f)
$$

for $t \in\left[t_{1}, b\right], m \geq 0$, which follow directly from Theorem 1 .

\subsection{More general boundary conditions}

With appropriate modifications, the approach is also applicable for other types of boundary conditions. If, for example, instead of the Dirichlet conditions (1.4), one considers the condition

$$
\phi\left(u(a), u^{\prime}(a), u(b), u^{\prime}(b)\right)=0
$$

with $\phi: \mathbb{R}^{4} \rightarrow \mathbb{R}^{2}$, then the approximate values of the parameters should be found from (2.38) complemented by the equations

$$
\phi\left(z_{1}, z_{2}, \lambda_{1}, \lambda_{2}\right)=0 .
$$

Relations (2.42) are deduced from the requirement that the iterations should satisfy the boundary conditions (2.41):

$$
\phi\left(x_{m, 1}(a, z, \eta), x_{m, 2}(a, z, \eta), y_{m, 1}(b, \eta, \lambda), y_{m, 2}(b, \eta, \lambda)\right)=0,
$$

whence, in view of the recurrence relations (2.19)-(2.22) and transformation of the second order equation (1.3) into (2.4), (2.5), one arrives at (2.42). Then (2.38), (2.42) is a system of six equations with the same number of unknowns.

\section{3. $\left(\sigma_{0}, \sigma_{1} ; t_{1}\right)$ SOLUTIONS OF THE DIRICHLET PROBLEM}

The argument from the preceding section can be adopted for finding solutions with prescribed number of zeroes. Let us explain the idea in application to the Dirichlet problem (1.3), (1.4).

Definition 1. Let $l \in \mathbb{N},\left\{\sigma_{0}, \sigma_{1}, \ldots, \sigma_{l}\right\} \subset\{-1,1\}$, and $t_{1}, \ldots, t_{l}$ are points such that $a<t_{1}<t_{2}<\cdots<t_{l}<b$. We say that a continuous function $u:[a, b] \rightarrow \mathbb{R}$ is of type $\left(\sigma_{0}, \sigma_{1}, \ldots, \sigma_{l} ; t_{1}, t_{2}, \ldots, t_{l}\right)$ if

$$
\sigma_{k-1} u(t)>0 \quad \text { for } t \in\left(t_{k-1}, t_{k}\right), k=1,2, \ldots, l+1,
$$

where $t_{0}:=a, t_{l+1}:=b$.

In other words, $u$ possessing this property has zero value at the points $t_{1}, \ldots, t_{l}$ and its sign before vanishing at $t_{k}$ is $\sigma_{k-1}, k=1,2, \ldots, l$. For $l=0$, it is natural to understand type $\left(\sigma_{0}\right)$ as the case where $u$ has no zeroes on $(a, b)$ and sign $u \equiv \sigma_{0}$.

In relation to problem $(1.3),(1.4)$, the property $\left(\sigma_{0}, \sigma_{1}, \ldots, \sigma_{l} ; t_{1}, t_{2}, \ldots, t_{l}\right)$, in fact, describes solutions with prescribed zeros and fixed signs around them. Here, we are interested in such solutions of equations similar to (1.2), (1.1) focusing on the case where $l=0$ or $l=1$, i.e., we will consider solutions of type $\left(\sigma_{0}\right)$ and $\left(\sigma_{0}, \sigma_{1} ; t_{1}\right)$. For 
$l=1$, a solution of problem (2.4)-(2.3) is composed from solutions of two auxiliary boundary value problems.

The techniques described above allow one to look for $\left(\sigma_{0}, \sigma_{1} ; t_{1}\right)$ solutions of the Dirichlet problem (1.3), (1.4) by rewriting it as (2.4), (2.5), (2.3), i.e., (2.1)-(2.3) with $f=\operatorname{col}\left(f_{1}, f_{2}\right)$ of the form

$$
f_{1}\left(t, u_{1}, u_{2}\right)=u_{2}, \quad f_{2}\left(t, u_{1}, u_{2}\right)=g\left(t, u_{1}, u_{2}\right)
$$

and studying the corresponding auxiliary problems on $\left[a, t_{1}\right]$ and $\left[t_{1}, b\right]$. Note that, for a $\left(\sigma_{0}, \sigma_{1} ; t_{1}\right)$ solution, we have $\operatorname{sign} u_{1}=\sigma_{0}$ on $\left[a, t_{1}\right]$ and $\operatorname{sign} u_{1}=\sigma_{1}$ on $\left[t_{1}, b\right]$; this fact can be used to simplify possible terms with absolute value signs in (3.1).

Suppose that we are looking for $\left(\sigma_{0}, \sigma_{1} ; t_{1}\right)$ solutions of problem (1.3), (1.4). The corresponding solution $u=\operatorname{col}\left(u_{1}, u_{2}\right)$ of (2.4), (2.5), (2.3) then has the property $u_{1}\left(t_{1}\right)=0$ and, therefore, Theorem 3 "detects" solutions $u$ with

$$
u(a)=\left(\begin{array}{c}
0 \\
z_{2}^{*}
\end{array}\right), \quad u\left(t_{1}\right)=\left(\begin{array}{c}
0 \\
\eta_{2}^{*}
\end{array}\right), \quad u(b)=\left(\begin{array}{c}
0 \\
\lambda_{2}^{*}
\end{array}\right),
$$

where $z_{2}^{*}, \eta_{2}^{*}, \lambda_{2}^{*}$, and $t_{1}$ are such that equalities (2.33) hold. Note that, with fixed $\eta_{1}=0$ and unknown $t_{1}$, the number of variables and equations in (2.33) is four, i. e., is the same as in Theorem 2. Thus, formally the same equations (2.33) which have been deduced in Section 2.4 for the parameters $\left(z_{2}, \eta_{1}, \eta_{2}, \lambda_{2}\right)$ serve in this case to determine the unknown values $\left(z_{2}, \eta_{2}, \lambda_{2}, t_{1}\right)$. We should only take into account different roles of $\eta_{1}$ and $t_{1}$ due to which, instead of (2.34), system (2.33) is now rewritten in the form

$$
\Delta^{0}\left(\left(\begin{array}{c}
0 \\
z_{2}
\end{array}\right),\left(\begin{array}{c}
0 \\
\eta_{2}
\end{array}\right)\right)=0, \quad \Delta^{1}\left(\left(\begin{array}{c}
0 \\
\eta_{2}
\end{array}\right),\left(\begin{array}{c}
0 \\
\lambda_{2}
\end{array}\right)\right)=0
$$

and involves the unknowns $\left(z_{2}, \eta_{2}, \lambda_{2}, t_{1}\right)$. The approximate values of the parameters $\left(z_{2}, \eta_{2}, \lambda_{2}, t_{1}\right)$ are looked for from the approximate determining equations

$$
\Delta_{m}^{0}\left(\left(\begin{array}{c}
0 \\
z_{2}
\end{array}\right),\left(\begin{array}{c}
0 \\
\eta_{2}
\end{array}\right)\right)=0, \quad \Delta_{m}^{1}\left(\left(\begin{array}{c}
0 \\
\eta_{2}
\end{array}\right),\left(\begin{array}{c}
0 \\
\lambda_{2}
\end{array}\right)\right)=0
$$

which, in view of (3.1), have the form

$$
\begin{gathered}
\int_{a}^{t_{1}} x_{m, 2}\left(s,\left(\begin{array}{c}
0 \\
z_{2}
\end{array}\right),\left(\begin{array}{c}
0 \\
\eta_{2}
\end{array}\right)\right) d s=0, \\
\int_{a}^{t_{1}} g\left(s, x_{m, 1}\left(s,\left(\begin{array}{c}
0 \\
z_{2}
\end{array}\right),\left(\begin{array}{c}
0 \\
\eta_{2}
\end{array}\right)\right), x_{m, 2}\left(s,\left(\begin{array}{c}
0 \\
z_{2}
\end{array}\right),\left(\begin{array}{c}
0 \\
\eta_{2}
\end{array}\right)\right)\right) d s=\eta_{2}-z_{2}, \\
\int_{t_{1}}^{b} y_{m, 2}\left(s,\left(\begin{array}{c}
0 \\
\eta_{2}
\end{array}\right),\left(\begin{array}{c}
0 \\
\lambda_{2}
\end{array}\right)\right) d s=0, \\
\int_{t_{1}}^{b} g\left(s, y_{m, 1}\left(s,\left(\begin{array}{c}
0 \\
\eta_{2}
\end{array}\right),\left(\begin{array}{c}
0 \\
\lambda_{2}
\end{array}\right)\right), y_{m, 2}\left(s,\left(\begin{array}{c}
0 \\
\eta_{2}
\end{array}\right),\left(\begin{array}{c}
0 \\
\lambda_{2}
\end{array}\right)\right)\right) d s=\lambda_{2}-\eta_{2} .
\end{gathered}
$$

Here, (3.5), (3.6) (resp., (3.7), (3.8)) correspond to the auxiliary problem on $\left[a, t_{1}\right]$ (resp., $\left.\left[t_{1}, b\right]\right)$. 


\section{COMPUTATION FOR CONCRETE EXAMPLES}

The technique described above can be efficiently used, in particular, for finding $\left(\sigma_{0}\right)$ and $\left(\sigma_{0}, \sigma_{1} ; t_{1}\right)$ solutions of Emden-Fowler type equations. In order to apply it to the Dirichlet problem (1.3), (1.4), we first rewrite (1.3) in the form (2.4), (2.5). The sets $\Omega_{0}\left(\rho_{0}\right), \Omega_{1}\left(\rho_{1}\right)$ are defined according to (2.18) with some fixed sets $D_{a}$, $D_{b}$ satisfying (2.17). It is not difficult to verify that the vectors $\rho_{0}, \rho_{1}$, according to (2.23), should satisfy the inequalities

$$
\rho_{0} \geq \frac{t_{1}-a}{4}\left(\begin{array}{l}
\operatorname{diampr}_{2} \Omega_{0}\left(\rho_{0}\right) \\
\delta_{\left[a, t_{1}\right], \Omega_{0}\left(\rho_{0}\right)}(g)
\end{array}\right), \quad \rho_{1} \geq \frac{b-t_{1}}{4}\left(\begin{array}{c}
\operatorname{diampr}_{2} \Omega_{1}\left(\rho_{1}\right) \\
\delta_{\left[t_{1}, b\right], \Omega_{1}\left(\rho_{1}\right)}(g)
\end{array}\right) .
$$

Here, for $\Omega \subset \mathbb{R}^{2}$ and $J \subset \mathbb{R}$, we use the notation $\operatorname{diam} J:=\sup J-\inf J$ and $\operatorname{pr}_{2} \Omega:=\left\{z_{2} \mid\left(z_{1}, z_{2}\right) \in \Omega\right.$ for some $\left.z_{1}\right\}$.

The Lipschitz condition from Theorem 1 in this case means that

$$
\left|g\left(t, u_{1}, u_{2}\right)-g\left(t, v_{1}, v_{2}\right)\right| \leq k_{i 1}\left|u_{1}-v_{1}\right|+k_{i 2}\left|u_{2}-v_{2}\right|
$$

for $t \in\left[(1-i) a+i b,(1-i) t_{1}+i b\right],\left\{u_{j}, v_{j} \mid j=1,2\right\} \subset \Omega_{i}\left(\rho_{i}\right), i=0,1$, with some positive constants $\left\{k_{i j} \mid i=0,1, j=1,2\right\}$ satisfying the inequalities

$$
k_{02}+\sqrt{k_{02}^{2}+4 k_{01}}<\frac{20}{3\left(t_{1}-a\right)}, \quad k_{12}+\sqrt{k_{12}^{2}+4 k_{11}}<\frac{20}{3\left(b-t_{1}\right)} .
$$

Thus, Assumptions 1-3 of Section 2 reformulated for problem (2.4), (2.5) consist in conditions (4.1)-(4.3). They mean that the function $g$ from (1.3) is Lipschitzian on a set which is sufficiently wide (existence of $\rho_{0}, \rho_{1}$ satisfying (4.1)) with constants which are sufficiently small (condition (4.3)). We omit the details of verification of these conditions in the examples below.

\subsection{Two sign-constant solutions of (4.4)-(4.6), $p=1$}

Consider the Dirichlet problem

$$
\begin{gathered}
u^{\prime \prime}(t)=-\gamma q(t) u^{3}(t), \quad t \in[a, b], \\
u(a)=0, \quad u(b)=0,
\end{gathered}
$$

where $a=-1, b=1$, and $q$ is a $\vee$-shaped piecewise linear function,

$$
q(t)= \begin{cases}\left(-\frac{2}{3} t+\frac{1}{3}\right)^{p} & \text { for } t \in[a, 0], \\ \left(\frac{2}{3} t+\frac{1}{3}\right)^{p} & \text { for } t \in[0, b],\end{cases}
$$

with $p=1, \gamma=2$. We will show by computation that this problem has two solutions (positive and negative, which are almost symmetric to one another). 
We take, e. g., $t_{1}=0^{2}$ and study the auxiliary boundary value problems (2.6)-(2.7) and (2.8)-(2.9) using the successive approximations (2.20), (2.22).

Constructing $x_{1}(\cdot, z, \eta)$ and $y_{1}(\cdot, \eta, \lambda)$ according to (2.20), (2.22) and solving the approximate algebraic determining system (2.38) for $m=1$, we obtain the following numerical values for the unknown parameters:

$$
\eta_{1} \approx 1.953587407, \quad \eta_{2} \approx 0, \quad \lambda_{2} \approx-2.995901364, \quad z_{2} \approx 2.995901364 .
$$

Inserting values (4.7) into the expressions for $x_{1}(\cdot, z, \eta)$ and $y_{1}(\cdot, \eta, \lambda)$, we obtain the first approximation (2.40) which has the form

$$
U_{1}(t)= \begin{cases}X_{1}(t)=\operatorname{col}\left(X_{1,1}(t), X_{1,2}(t)\right) & \text { for } t \in\left[a, t_{1}\right], \\ Y_{1}(t)=\operatorname{col}\left(Y_{1,1}(t), Y_{1,2}(t)\right) & \text { for } t \in[a, b],\end{cases}
$$

with

$$
\begin{aligned}
X_{1,1}(t) \approx & 1.953587407+0.455636725 t-1.497950682 t^{2}, \\
X_{1,2}(t) \approx & 1.988232981 t^{5}+6.213228065 t^{4}+4.970582452 t^{3} \\
& -2.485291226 t^{2}-6.226779958 t
\end{aligned}
$$

for $t \in\left[a, t_{1}\right]$ and

$$
\begin{aligned}
Y_{1,1}(t) \approx & 1.953587407-0.455636725 t-1.497950682 t^{2}, \\
Y_{1,2}(t) \approx & 1.988232981 t^{5}-6.213228065 t^{4}+4.970582452 t^{3} \\
& +2.485291226 t^{2}-6.226779958 t
\end{aligned}
$$

for $t \in\left[t_{1}, b\right]$.

Higher approximations $(m=2,3, \ldots)$ are obtained by analogy to $(4.8)$. Note that the most difficult part is the construction of the functions $x_{m}(\cdot, z, \eta)$ and $y_{m}(\cdot, \eta, \lambda)$ in (2.20) and (2.22). If the explicit integration impossible or difficult, one can use suitable modifications of formulae (2.20) and (2.22) which, at the expense of a certain loss in accuracy, lead one to iterations better suited for practical computations.

In [19], we mention two natural modifications of this kind, namely the version of "frozen" parameters and the version with polynomial interpolation used in [16]. Let us apply here the "frozen" parameters version, which means that the approximations $X_{m}$ and $Y_{m}$ obtained on the $m$ th step are used directly instead of $x_{m}(\cdot, z, \eta)$ and $y_{m}(\cdot, \eta, \lambda)$ (i. e., the roots of the $m$ th determining system are substituted into the corresponding expressions before passing to the next step of iteration).

In our case, to obtain the second parametrized iterations (denote these functions by $\hat{x}_{2}(\cdot, z, \eta), \hat{y}_{2}(\cdot, \eta, \lambda)$ to distinguish them from those given by $\left.(2.20),(2.22)\right)$, instead

\footnotetext{
${ }^{2}$ In this case, the value of $t_{1}$ can be chosen directly since sign-constant solutions are looked for and, thus, $t_{1}$ does not have the meaning of a zero of the solution. The choice $t_{1}=0$ is also natural because of the form of the forcing term (4.6).
} 
of $x_{1}(\cdot, z, \eta)$ and $y_{1}(\cdot, \eta, \lambda)$, we use the respective components of $(4.8)$ :

$$
\begin{aligned}
\hat{x}_{2}(t, z, \eta)= & \int_{a}^{t} f\left(s, X_{1,1}(s), X_{1,2}(s)\right) d s-\frac{t-a}{t_{1}-a} \int_{a}^{t_{1}} f\left(s, X_{1,1}(s), X_{1,2}(s)\right) d s \\
& +z+\frac{t-a}{t_{1}-a}(\eta-z), \quad t \in\left[a, t_{1}\right], \\
\hat{y}_{2}(t, \eta, \lambda)= & \int_{t_{1}}^{t} f\left(s, Y_{1,1}(s), Y_{1,2}(s)\right) d s-\frac{t-t_{1}}{b-t_{1}} \int_{t_{1}}^{b} f\left(s, Y_{1,1}(s), Y_{1,2}(s)\right) d s+ \\
& +\eta+\frac{t-t_{1}}{b-t_{1}}(\lambda-\eta), \quad t \in\left[t_{1}, b\right] .
\end{aligned}
$$

Then we need to solve the second approximate determining system (2.38) which, for $m=2$, has the form

$$
\eta-z=\int_{a}^{t_{1}} f\left(s, X_{11}(s), X_{12}(s)\right) d s, \quad \lambda-\eta=\int_{t_{1}}^{b} f\left(s, Y_{11}(s), Y_{12}(s)\right) d s .
$$

Note that the right-hand side terms of the latter system does not involve $z, \eta$, and $\lambda$, which is a major simplification. For $m>2$, approximations $X_{m}, Y_{m}$ are obtained by analogy using $\hat{x}_{m}(\cdot, z, \eta), \hat{y}_{m}(\cdot, \eta, \lambda)$ constructed as above.

For our problem, carrying out computations by the "frozen" technique and solving the respective equations, we obtain the parameter values presented in Table 1.

\begin{tabular}{clccc}
\hline$m$ & \multicolumn{1}{c}{$\eta_{1}$} & $\eta_{2}$ & $\lambda_{2}$ & $z_{2}$ \\
\hline 3 & 1.851388676 & $3.483457908 \cdot 10^{-10}$ & -2.622412592 & 2.622412592 \\
4 & 1.8557702 & $1.295121405 \cdot 10^{-9}$ & -2.613402579 & 2.613402576 \\
5 & 1.857259119 & $-1.810452349 \cdot 10^{-9}$ & -2.617838558 & 2.617838555 \\
7 & 1.856920628 & $-1.173443333 \cdot 10^{-9}$ & -2.613691252 & 2.613691240 \\
\hline
\end{tabular}

TABLE 1.

Computing the residual for the approximate (positive) solution $U_{7,1}$, we find that it does not exceeed 0.0005. The graph of $U_{7,1}$ is shown on Figure 1 .

According to Theorems 2 and 3, the number of solutions of the problem under consideration with the graphs lying in the given region is equal to the number of roots of the determining system (2.33). A computation shows that the approximate determining system (2.38) for $m=1$, along with (4.7), has another root

$$
\eta_{1} \approx-1.953587407, \quad \eta_{2} \approx 0, \quad \lambda_{2} \approx 2.995901364, \quad z_{2} \approx-2.995901364,
$$

which indicates the presence of another (negative) solution of the problem. Refining these values on the next steps by using the "frozen" technique, we obtain the approximate solution corresponding to the parameter values shown in Table 2. 


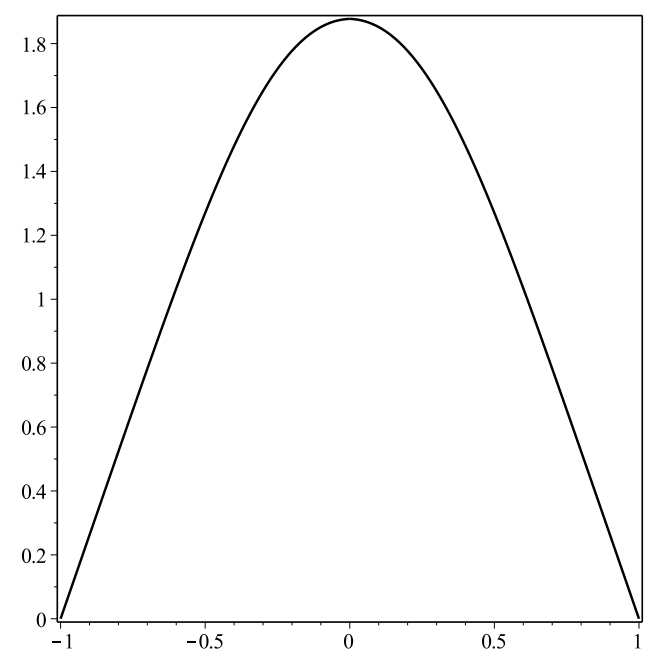

FIGURE 1. The seventh approximation to the positive solution.

\begin{tabular}{clccc}
\hline$m$ & \multicolumn{1}{c}{$\eta_{1}$} & $\eta_{2}$ & $\lambda_{2}$ & $z_{2}$ \\
\hline 4 & -1.862639362 & $-3.695522622 \cdot 10^{-9}$ & 2.631889316 & -2.631889323 \\
5 & -1.859693541 & $1.253485922 \cdot 10^{-9}$ & 2.620422812 & -2.620422815 \\
7 & -1.8571756 & $-2.953304145 \cdot 10^{-9}$ & 2.613550388 & -2.613550391 \\
8 & -1.856620650 & $-1.408647961 \cdot 10^{-10}$ & 2.612670399 & -2.612670398 \\
\hline
\end{tabular}

TABLE 2.

The residual for the approximate (negative) solution $U_{8,1}$ does not exceed 0.001 . The graph of $U_{8,1}$ is shown on Figure 3.

4.2. Two sign-constant solutions of (4.4)-(4.6), $p=1 / 3$

Consider the Dirichlet problem (4.4)-(4.6) for $a=-1, b=1, p=1 / 3, \gamma=2$. In this case we also obtain two solutions (positive and negative ones).

\begin{tabular}{ccccc}
\hline$m$ & $\eta_{1}$ & $\eta_{2}$ & $\lambda_{2}$ & $z_{2}$ \\
\hline 1 & 1.543922367 & 0 & -2.270535575 & 2.270535575 \\
2 & 1.516958742 & $-1.438012983 \cdot 10^{-12}$ & -2.062927555 & 2.062927555 \\
3 & 1.484912528 & $1.783555583 \cdot 10^{-10}$ & -2.017809966 & 2.017809966 \\
\hline
\end{tabular}

TABLE 3. 


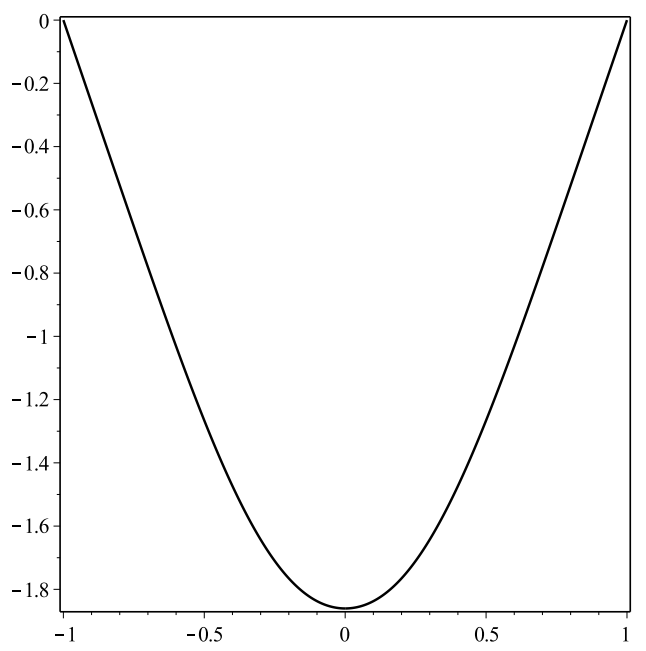

FIGURE 2. The eighth approximation to the negative solution.

The results of computation using formulae (2.20) and (2.22) (without "frozen" technique) for several steps of iteration are given in Table 3. The corresponding approximation positive solution $U_{3,1}(m=3)$ is shown on Figure 3. The residual for $U_{3,1}$ does not exceed 0.02 .

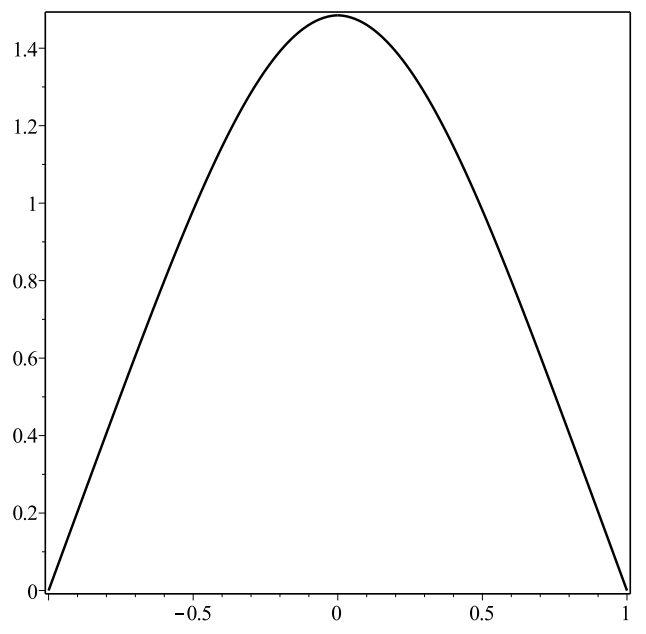

FIGURE 3. The third approximation to the positive solution. 
The approximate negative solution (which appears to be symmetric to the positive one) is specified by the roots of the approximate determining equations (without frozen technique) shown in Table 4 . The residual of this approximate solution for $m=3$ does not exceed 0.025, its graph is shown on Figure 4 .

\begin{tabular}{|c|c|c|c|c|}
\hline$m$ & $\eta_{1}$ & $\eta_{2}$ & $\lambda_{2}$ & $z_{2}$ \\
\hline 1 & -1.543922367 & 0 & 2.270535575 & -2.2705355756 \\
\hline 3 & -1.484912528 & $2.569148168 \cdot 10^{-10}$ & 2.017809966 & -2.017809967 \\
\hline
\end{tabular}

TABLE 4.

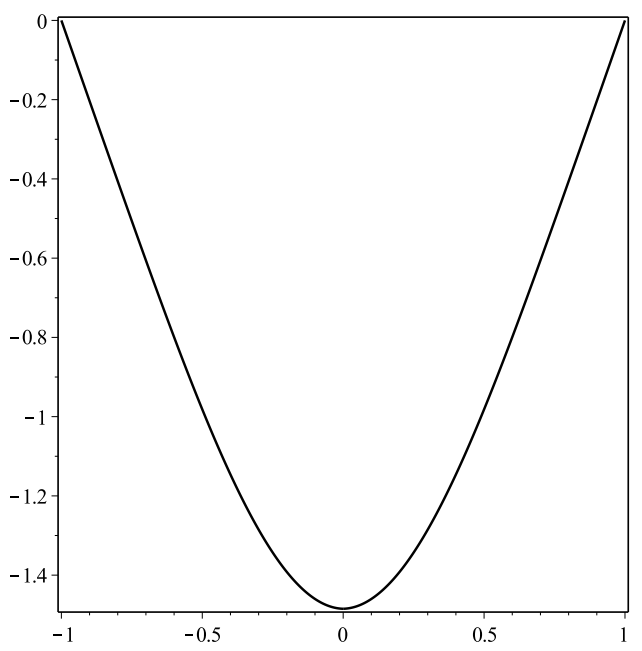

FIGURE 4. The third approximation to the negative solution.

4.3. $\left(-1,1 ; t_{1}\right)$ solution of (4.9) with $a=-1 / 2, b=1 / 2$, and the Dirichlet conditions (4.10)

Consider the Dirichlet problem

$$
\begin{gathered}
u^{\prime \prime}(t)=-\frac{1}{3}\left(8 t^{2}+1\right)|u(t)|^{3} u(t)+r(t), \quad t \in(a, b), \\
u(a)=0, \quad u(b)=0
\end{gathered}
$$

with $a=-1 / 2, b=1 / 2$, and

$$
r(t)= \begin{cases}2-\frac{1}{3}\left(8 t^{2}+1\right)\left(t^{2}+\frac{1}{4} t-\frac{1}{8}\right)^{4} & \text { for } t \in\left[-\frac{1}{2}, t_{1}\right], \\ -6+\frac{1}{3}\left(8 t^{2}+1\right)\left(-3 t^{2}+\frac{9}{4} t-\frac{3}{8}\right)^{4} & \text { for } t \in\left[t_{1}, \frac{1}{2}\right],\end{cases}
$$


where $t_{1}$ is a point from $(-1 / 2,1 / 2)$. Let us focus on $\left(-1,1 ; t_{1}\right)$ solutions of problem (4.9), (4.10). According to the definition from Section 3, this means that the solution $u$ should be negative on $\left(a, t_{1}\right)$, positive on $\left(t_{1}, b\right)$, and zero at $t_{1}$. The value of $t_{1} \in(a, b)$ is a priori unknown and has to be determined.

Let us proceed as described in Section 3. We pass to the corresponding problem (2.4), (2.5), (2.3) by rewriting (4.9) in the form of system (2.4), (2.5) with

$$
g\left(t, u_{1}, u_{2}\right):=-\frac{1}{3}\left(8 t^{2}+1\right)\left|u_{1}\right|^{3} u_{1}+r(t) .
$$

Since we focus on $\left(-1,1 ; t_{1}\right)$ solutions, $(4.12)$ can be replaced by the formula

$$
g\left(t, u_{1}, u_{2}\right)=r(t)+ \begin{cases}\frac{1}{3}\left(8 t^{2}+1\right) u_{1}^{4} & \text { if } t \in\left[-\frac{1}{2}, t_{1}\right] \\ -\frac{1}{3}\left(8 t^{2}+1\right) u_{1}^{4} & \text { if } t \in\left[t_{1}, \frac{1}{2}\right]\end{cases}
$$

A $\left(-1,1 ; t_{1}\right)$ solution of (4.9), (4.10) is composed from solutions $x, y$ of the auxiliary boundary value problems (2.6), (2.7) and (2.8), (2.9) for $f=\operatorname{col}\left(f_{1}, f_{2}\right)$ of form (3.1) with $g$ given by (4.13). For the auxiliary problems, the corresponding iteration sequences $\left\{x_{m}(\cdot, z, \eta) \mid m \geq 0\right\}$ and $\left\{y_{m}(\cdot, \eta, \lambda) \mid m \geq 0\right\}$ are constructed according to (2.19)-(2.22). Note that the functions depend on the unknown $t_{1}$.

Computing the functions $x_{1}\left(\cdot,\left(\begin{array}{c}0 \\ z_{2}\end{array}\right),\left(\begin{array}{c}0 \\ \eta_{2}\end{array}\right)\right), y_{1}\left(\cdot,\left(\begin{array}{c}0 \\ \eta_{2}\end{array}\right),\left(\begin{array}{c}0 \\ \lambda_{2}\end{array}\right)\right)$ according to (2.20), (2.22) for $m=1$ and solving the first approximate determining system (3.5)-(3.8), we find the numerical values of the unknown parameters shown in Table 5.

\begin{tabular}{ccccc}
\hline$m$ & $\eta_{2}$ & $\lambda_{2}$ & $t_{1}$ & $z_{2}$ \\
\hline 1 & 0.7500010172 & -0.7500010060 & 0.2499996629 & -0.7499983085 \\
2 & 0.7499999991 & -0.7499999996 & 0.2500000003 & -0.7499999990 \\
3 & 0.7500000001 & -0.7500000006 & 0.2500000000 & -0.7499999999 \\
\hline
\end{tabular}

TABLE 5 .

The values from Table 5 determine the corresponding approximate solutions (2.40). Note that, as is easy to verify, the function

$$
u^{*}(t)= \begin{cases}t^{2}+\frac{1}{4} t-\frac{1}{8} & \text { for } t \in\left[-\frac{1}{2}, \frac{1}{4}\right] \\ -3 t^{2}+\frac{9}{4} t-\frac{3}{8} & \text { for } t \in\left[\frac{1}{4}, \frac{1}{2}\right]\end{cases}
$$

is an exact solution of the boundary value problem (4.9), (4.10). Clearly, solution (4.14) is of type $(-1,1 ; 1 / 4)$.

The graph of the exact solution (4.14) and the approximate $\left(-1,1 ; t_{1}\right)$ solution for $m=2$ are shown on the Figure 5. The residual of the approximate solution is about $4 \cdot 10^{-9}$. 


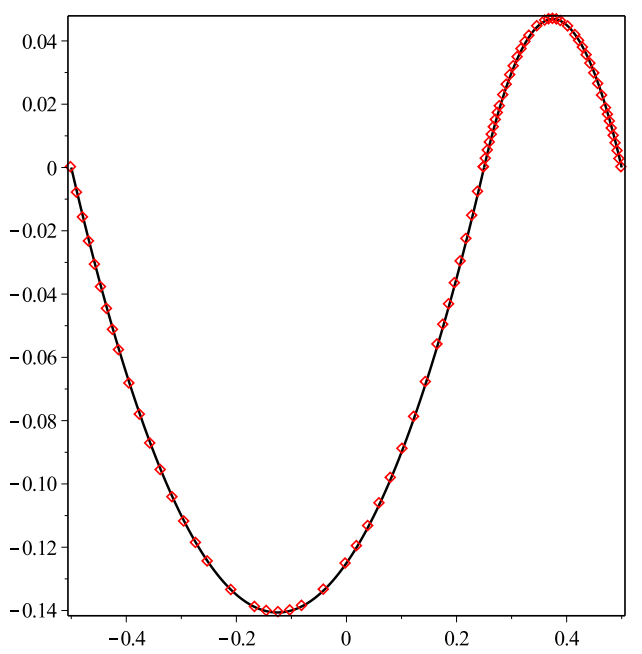

FIGURE 5. The exact solution (4.14) and its second approximation

4.4. $\left(1,-1 ; t_{1}\right)$ solution of $(4.9)$ with the non-linear two-point conditions (4.16) and $b=1 / 4$

Consider equation (4.9) with $a=-1 / 2, b=1 / 4$,

$$
r(t):= \begin{cases}2+\left(\frac{8}{3} t^{2}+\frac{1}{3}\right)\left(t^{2}-\frac{1}{12} t-\frac{1}{12}\right)^{4} & \text { if } t \in\left[-\frac{1}{2}, t_{1}\right], \\ 2-\left(\frac{8}{3} t^{2}+\frac{1}{3}\right)\left(t^{2}-\frac{1}{12} t-\frac{1}{12}\right)^{4} & \text { if } t \in\left[t_{1}, \frac{1}{4}\right],\end{cases}
$$

where $t_{1}$ is an unknown point from $(-1 / 2,1 / 4)$. Let us look for $\left(1,-1 ; t_{1}\right)$ solutions of this equation satisfying the two-point boundary conditions

$$
u\left(-\frac{1}{2}\right) u^{\prime}\left(\frac{1}{4}\right)=\frac{25}{288}, \quad u^{\prime}\left(-\frac{1}{2}\right)+u^{\prime}\left(\frac{1}{4}\right)=-\frac{2}{3} .
$$

Similarly to Section 4.3, we rewrite equation (4.9) as system (2.4), (2.5) with

$$
g\left(t, u_{1}, u_{2}\right)=r(t)+ \begin{cases}-\frac{1}{3}\left(8 t^{2}+1\right) u_{1}^{4} & \text { if } t \in\left[-\frac{1}{2}, t_{1}\right], \\ \frac{1}{3}\left(8 t^{2}+1\right) u_{1}^{4} & \text { if } t \in\left[t_{1}, \frac{1}{4}\right]\end{cases}
$$

and consider (2.4), (2.5) under the boundary conditions

$$
u_{1}\left(-\frac{1}{2}\right) u_{2}\left(\frac{1}{4}\right)=\frac{25}{288}, \quad u_{2}\left(-\frac{1}{2}\right)+u_{2}\left(\frac{1}{4}\right)=-\frac{2}{3} \text {. }
$$

In (4.17), we have used the information on the sign of $u_{1}$ contained in the $\left(1,-1 ; t_{1}\right)$ property. 
Having constructed the functions $x_{1}\left(\cdot,\left(\begin{array}{c}z_{1} \\ z_{2}\end{array}\right),\left(\begin{array}{c}0 \\ \eta_{2}\end{array}\right)\right)$ and $y_{1}\left(\cdot,\left(\begin{array}{c}0 \\ \eta_{2}\end{array}\right),\left(\begin{array}{c}\lambda_{1} \\ \lambda_{2}\end{array}\right)\right)$ according to (2.20) and (2.22), we write the corresponding equations (2.38):

$$
\Delta_{m}^{0}\left(\left(\begin{array}{c}
z_{1} \\
z_{2}
\end{array}\right),\left(\begin{array}{c}
0 \\
\eta_{2}
\end{array}\right)\right)=0, \quad \Delta_{m}^{1}\left(\left(\begin{array}{c}
0 \\
\eta_{2}
\end{array}\right),\left(\begin{array}{c}
\lambda_{1} \\
\lambda_{2}
\end{array}\right)\right)=0
$$

with $m=1$. Note that, in this case, we look for the values of $\left(z_{1}, z_{2}, \eta_{2}, \lambda_{1}, \lambda_{2}, t_{1}\right)$ since no a priori information the values of $u_{1}$ at $a$ and $b$ is available.

Recalling Section 2.6 and complementing (2.37) by the equations

$$
z_{1} \lambda_{2}=\frac{25}{288}, \quad z_{2}+\lambda_{2}=-\frac{2}{3}
$$

generated by the boundary conditions $(4.18)^{3}$, we get a system of six equations with six unknowns. Solving (4.19), (4.20) with $m=1$, we obtain the first approximation to the values of the unknown parameters. Repeating the same for $m=2,3$, we arrive at Table 6.

\begin{tabular}{cccc}
\hline & $m=1$ & $m=2$ & $m=3$ \\
\hline$z_{1}$ & 0.2083355451 & 0.2083355451 & 0.2083355451 \\
$z_{2}$ & -1.083328910 & -1.083328910 & -1.083328910 \\
$\eta_{2}$ & -0.5833204107 & -0.58331754047 & -0.5833175405 \\
$\lambda_{1}$ & -0.04166396646 & -0.04166295247 & -0.04166295248 \\
$\lambda_{2}$ & 0.416662243 & 0.4166622431 & 0.4166622432 \\
$t_{1}$ & -0.2499957487 & -0.2499943135 & -0.2499943135 \\
\hline
\end{tabular}

TABLE 6.

One can check that the function

$$
u(t)=t^{2}-\frac{t+1}{12}, \quad t \in\left[-\frac{1}{2}, \frac{1}{4}\right],
$$

is an exact $\left(1,-1 ;-\frac{1}{4}\right)$ solution of our problem (4.9), (4.16). The graphs of (4.21) and the approximate solution generated by the values of parameters from Table 6 $(m=3)$ are presented on Figure 6. The residual of the approximate solution is about $6 \cdot 10^{-10}$.

4.5. $\left(1,-1 ; t_{1}\right)$ solution of $(4.9)$ with two-point conditions (4.22) and $b=1 / 4$

Let us look for a $\left(1,-1 ; t_{1}\right)$ solution of equation (4.9) with $a=-1 / 2, b=1 / 4$, and $r$ given by (4.15) (see Section 4.4) under the boundary conditions

$$
u\left(-\frac{1}{2}\right) u^{\prime}\left(\frac{1}{4}\right)=\frac{25}{288}, \quad\left|u\left(\frac{1}{4}\right)\right|=\frac{1}{24} .
$$

\footnotetext{
${ }^{3}$ We should recall the meaning of the parameters involved in (2.19)-(2.22); see (2.7), (2.9).
} 


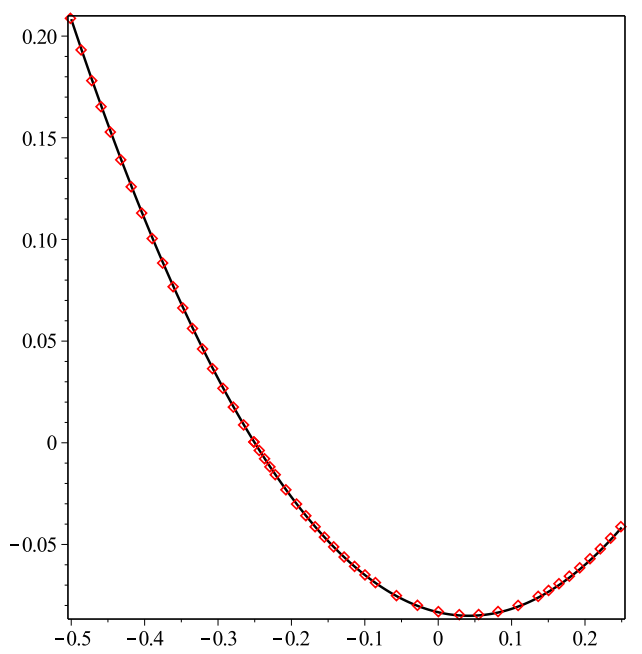

FIGURE 6. The exact solution (4.21) and its third approximation.

The approximate determining system consists in (2.38) and two additional equations (see Section 2.6)

$$
z_{1} z_{2}=\frac{25}{288}, \quad\left|\lambda_{1}\right|=\frac{1}{24}
$$

Solving system (2.38), (4.23) for $m=1,2,3$, we obtain the numerical values of parameters given in Table 7. Note that the exact solution of this problem has form (4.21);

\begin{tabular}{cccc}
\hline & $m=1$ & \multicolumn{1}{c}{$m=2$} & \multicolumn{1}{c}{$m=3$} \\
\hline$z_{1}$ & 0.2083409826 & 0.2083429898 & 0.2083429758 \\
$z_{2}$ & -1.083339776 & -1.083343787 & -1.083343759 \\
$\eta_{2}$ & -0.5833219625 & -0.5833196642 & -0.5833196604 \\
$\lambda_{1}$ & -0.04166668456 & -0.04166667360 & -0.04166666666 \\
$\lambda_{2}$ & 0.4166513687 & 0.416647354 & 0.4166473826 \\
$t_{1}$ & -0.2499910875 & -0.249987931 & -0.2499879432 \\
\hline
\end{tabular}

TABLE 7.

we see that the last row in the table gives approximate values of the exact value $t_{1}=-1 / 4$.

The graphs of the exact and approximate $\left(1,-1 ; t_{1}\right)$ solutions of problem (4.9), (4.22) generated by the values of parameters from Table 7 for $m=3$ practically coincide with those presented on Figure 6 . The residual of the approximate solution in this case is about $3 \cdot 10^{-10}$. 


\section{REFERENCES}

[1] V. I. Astashova and V. V. Rogachev, "On the number of zeros of oscillating solutions of the third- and fourth-order equations with power nonlinearities," J. Math. Sci. (N.Y.), vol. 205, no. 6, pp. 733-748, 2015, doi: 10.1007/s10958-015-2279-7. [Online]. Available: http://dx.doi.org/10.1007/s10958-015-2279-7

[2] A. Capietto, M. Henrard, J. Mawhin, and F. Zanolin, "A continuation approach to some forced superlinear Sturm-Liouville boundary value problems," Topol. Methods Nonlinear Anal., vol. 3, no. 1, pp. 81-100, 1994.

[3] A. Capietto, J. Mawhin, and F. Zanolin, "On the existence of two solutions with a prescribed number of zeros for a superlinear two-point boundary value problem," Topol. Methods Nonlinear Anal., vol. 6, no. 1, pp. 175-188, 1995.

[4] A. Gritsans and F. Sadyrbaev, "Characteristic numbers of non-autonomous Emden-Fowler type equations," Math. Model. Anal., vol. 11, no. 3, pp. 243-252, 2006.

[5] R. Kajikiya, "Non-even least energy solutions of the Emden-Fowler equation," Proc. Amer. Math. Soc., vol. 140, no. 4, pp. 1353-1362, 2012, doi: 10.1090/S0002-9939-2011-11172-9. [Online]. Available: http://dx.doi.org/10.1090/S0002-9939-2011-11172-9

[6] R. Kajikiya, "Non-even positive solutions of the Emden-Fowler equations with signchanging weights," Proc. Roy. Soc. Edinburgh Sect. A, vol. 143, no. 3, pp. 631-642, 2013, doi: 10.1017/S0308210511001594. [Online]. Available: http://dx.doi.org/10.1017/ S0308210511001594

[7] I. T. Kiguradze and T. A. Chanturia, Asymptotic properties of solutions of nonautonomous ordinary differential equations, ser. Mathematics and its Applications (Soviet Series). Kluwer Academic Publishers Group, Dordrecht, 1993, vol. 89, translated from the 1985 Russian original. [Online]. Available: http://dx.doi.org/10.1007/978-94-011-1808-8. doi: 10.1007/978-94-0111808-8

[8] J. Mawhin, Topological degree methods in nonlinear boundary value problems, ser. CBMS Regional Conference Series in Mathematics. American Mathematical Society, Providence, R.I., 1979, vol. 40, expository lectures from the CBMS Regional Conference held at Harvey Mudd College, Claremont, Calif., June 9-15, 1977.

[9] K. McLeod, W. C. Troy, and F. B. Weissler, "Radial solutions of $\delta u+f(u)=0$ with prescribed numbers of zeros," J. Differ. Equations, vol. 83, no. 2, pp. 368-378, 1990, doi: 10.1016/00220396(90)90063-U.

[10] R. A. Moore and Z. Nehari, "Nonoscillation theorems for a class of nonlinear differential equations," Trans. Amer. Math. Soc., vol. 93, pp. 30-52, 1959, doi: 10.2307/1993421.

[11] M. Naito, "On the number of zeros of bounded nonoscillatory solutions to higher-order nonlinear ordinary differential equations," Arch. Math. (Brno), vol. 43, no. 1, pp. 39-53, 2007.

[12] M. Naito and Y. Naito, "Solutions with prescribed numbers of zeros for nonlinear second order differential equations," Funkcial. Ekvac., vol. 37, no. 3, pp. 505-520, 1994. [Online]. Available: http://www.math.kobe-u.ac.jp/ fe/xml/mr1311557.xml

[13] Y. Naito, "Bounded solutions with prescribed numbers of zeros for the Emden-Fowler differential equation," Hiroshima Math. J., vol. 24, no. 1, pp. 177-220, 1994. [Online]. Available: http://projecteuclid.org/euclid.hmj/1206128140

[14] Z. Nehari, "Characteristic values associated with a class of non-linear second-order differential equations," Acta Math., vol. 105, pp. 141-175, 1961, doi: 10.1007/BF02559588. [Online]. Available: http://dx.doi.org/10.1007/BF02559588 
[15] A. Rontó and M. Rontó, "Successive approximation techniques in non-linear boundary value problems for ordinary differential equations," in Handbook of differential equations: ordinary differential equations. Vol. IV, ser. Handb. Differ. Equ. Elsevier/North-Holland, Amsterdam, 2008, pp. 441-592, doi: 10.1016/S1874-5725(08)80010-7.

[16] A. Rontó, M. Rontó, G. Holubová, and P. Nečesal, "Numerical-analytic technique for investigation of solutions of some nonlinear equations with Dirichlet conditions," Bound. Value Probl., pp. 2011:58, 20, 2011, doi: 10.1186/1687-2770-2011-58. [Online]. Available: http://dx.doi.org/10.1186/1687-2770-2011-58

[17] A. Rontó, M. Rontó, and N. Shchobak, "Constructive analysis of periodic solutions with interval halving," Bound. Value Probl., pp. 2013:57, 34, 2013, doi: 10.1186/1687-2770-2013-57. [Online]. Available: http://dx.doi.org/10.1186/1687-2770-2013-57

[18] A. Rontó, M. Rontó, and N. Shchobak, "Notes on interval halving procedure for periodic and twopoint problems,” Bound. Value Probl., pp. 2014:164, 20, 2014, doi: 10.1186/s13661-014-0164-9. [Online]. Available: http://dx.doi.org/10.1186/s13661-014-0164-9

[19] A. Rontó, M. Rontó, and J. Varha, "A new approach to non-local boundary value problems for ordinary differential systems," Appl. Math. Comput., vol. 250, pp. 689-700, 2015, doi: 10.1016/j.amc.2014.11.021. [Online]. Available: http://dx.doi.org/10.1016/j.amc.2014.11.021

[20] M. Rontó and Y. Varha, "Constructive existence analysis of solutions of non-linear integral boundary value problems," Miskolc Math. Notes, vol. 15, no. 2, pp. 725-742, 2014.

[21] M. Rontó and Y. Varha, "Successive approximations and interval halving for integral boundary value problems," Miskolc Math. Notes, vol. 16, no. 2, pp. 1129-1152, 2015, doi: 10.18514/MMN.2015.1708. [Online]. Available: http://dx.doi.org/10.18514/MMN.2015.1708

[22] M. Rontó, Y. Varha, and K. Marynets, "Further results on the investigation of solutions of integral boundary value problems," Tatra Mt. Math. Publ., vol. 63, pp. 247-267, 2015, doi: 10.1515/tmmp-2015-0036. [Online]. Available: http://dx.doi.org/10.1515/tmmp-2015-0036

\section{Authors' addresses}

\section{A. Rontó}

Institute of Mathematics, Czech Academy of Sciences, Zizkova 22, 61662 Brno, Czech Republic E-mail address: ronto@math.cas.cz

\section{Rontó}

Institute of Mathematics, University of Miskolc, 3515 Miskolc-Egyetemváros

E-mail address: matronto@uni-miskolc.hu

\section{N. Shchobak}

Brno University of Technology, Faculty of Business and Management, Kolejní 2906/4, 61200 Brno, Czech Republic

Current address: Uzhhorod National University, 14 Universytetska St., 88000, Uzhhorod, Ukraine 